\title{
Diffraction dissociation contributions to two-nucleon knockout reactions and the suppression of shell-model strength
}

\author{
J. A. Tostevin* \\ Department of Physics, School of Electronics and Physical Sciences, University of Surrey, Guildford, Surrey GU2 7XH, United Kingdom and \\ National Superconducting Cyclotron Laboratory, Michigan State University, East Lansing, Michigan 48824, USA
}

B. A. Brown

National Superconducting Cyclotron Laboratory, Michigan State University, East Lansing, Michigan 48824, USA and

Department of Physics and Astronomy, Michigan State University, East Lansing, Michigan 48824, USA

(Received 29 August 2006; published 11 December 2006)

\begin{abstract}
The contributions to the cross sections of intermediate energy two-nucleon knockout reactions from events in which one nucleon is removed by the stripping (inelastic breakup) mechanism and a second by the diffraction (elastic breakup) mechanism are discussed. The small additional contributions from two-nucleon diffraction events are also estimated. The approach used combines the eikonal reaction and shell model structure theory frameworks. For reactions involving the removal of two well-bound like nucleons, at incident energies of order $100 \mathrm{MeV}$ per nucleon, the additional cross sections are shown to be of approximately the same size as those from events in which both nucleons are stripped in inelastic interactions. These more complete dynamical calculations now permit a quantitative comparison of the theoretical cross sections with recent partial cross-section measurements of the two-neutron (two-proton) removal reactions from neutron-deficient (neutron-rich) nuclei. As has been observed in both nuclear- and electron-induced single-nucleon knockout reaction analyses, the theoretical two-nucleon knockout cross sections overestimate the measured values, requiring a suppression of the two nucleon shell-model transition strengths. The deduced two-nucleon suppression factors, $R_{s}(2 N)$, are consistent with a value of 0.5 for each of the five reactions considered.
\end{abstract}

DOI: 10.1103/PhysRevC.74.064604

PACS number(s): 21.10.Jx, 24.10.-i, 25.60.Gc, 27.30.+t

\section{INTRODUCTION}

Among the reactions that result in the removal of two like nucleons from a nucleus are (a) light-ion induced twonucleon transfer reactions, such as the $(p, t)$ reaction, e.g., Ref. [1]; (b) the ( $\left.e, e^{\prime} p p\right)$, electron-induced two-proton knockout reaction, [2] and references therein; and more recently (c) two-proton decay of nuclei near the proton dripline, e.g., Ref. [3] and references therein. All of these manifest sensitivity to the pairing interaction between like nucleons. They also reveal sensitivity of the associated transition strengths to the coherence of the two-nucleon configurations and to the model space used to describe the two-nucleon structures. Very recently, first measurements have also been made of the two-nucleon knockout reaction from exotic secondary fragmentation beams by a light nuclear target. The reactions probe the same two-nucleon transition densities that enter into the other reaction descriptions, but (i) in distinct regions of the nuclear chart and (ii) with different spin and spatial sensitivity to the two-nucleon wave function. The reactions we consider here involve the sudden, direct removal of pairs of like nucleons of a deficient nucleon species from some of the most exotic nuclei.

The two-proton removal reaction, from an intermediate energy beam of neutron-rich projectile nuclei, has recently been shown to proceed as a sudden direct reaction process $[4,5]$. Three new measurements, of two-neutron removal

\footnotetext{
*Electronic address: j.tostevin@surrey.ac.uk
}

reactions from the neutron-deficient $s d$-shell nuclei ${ }^{26} \mathrm{Si},{ }^{30} \mathrm{~S}$, and ${ }^{34} \mathrm{Ar}$, discussed theoretically in Ref. [5], were also recently reported [6]. These data, together with those of the two-proton removal reaction from ${ }^{28} \mathrm{Mg}$ [4], offer excellent test cases with which to assess the two-nucleon knockout theory on $s d$-shell nuclei: cases where shell-model structure predictions are expected to be very reliable.

The description of the structure of the two-removednucleons, and also that of the assumed direct reaction mechanism, were treated only very approximately in Ref. [4]. A more complete calculational scheme was subsequently presented in Ref. [5] and dealt with that part of the twonucleon removal cross section that arises from the stripping reaction mechanism, also referred to as inelastic breakup. That is, both of the removed nucleons were assumed to have interacted inelastically with the target nucleus. Furthermore, the theoretical approach of Ref. [5] showed how to combine fully the two-nucleon shell-model transition densities, and their associated spectroscopic amplitudes, with eikonal direct reaction theory. This analysis provided further evidence for the direct nature of the reaction mechanism in such systems. That there are significant differences between the spin selectivity of the knockout reaction mechanism and that of the light-ion twonucleon transfer reactions was also discussed in Ref. [5]. An initial discussion of the sensitivity of the two-nucleon knockout reaction to the spatial correlations of the two nucleons can be found in Ref. [7].

In the present article the earlier theoretical two-nucleon removal (stripping) cross section calculations [5] are developed further to include a full calculation of contributions to the cross 
section from events in which only one nucleon is absorbed (stripped) while a second is removed from the projectile by an elastic collision with the target (also called diffraction dissociation). Only an estimate of the (much smaller) cross section due to the removal of both of the well-bound nucleons by the elastic dissociation mechanism is included. The theoretical inclusive and partial cross predictions from the reaction theory and shell-model transition densities are compared with the relevant available data. Preliminary results of such model calculations have been reported elsewhere, being applied to direct two-proton knockout from ${ }^{44} \mathrm{~S}$ [8] and ${ }^{54} \mathrm{Ti}$ [9] and heavy nuclei [10] and to two-neutron knockout from ${ }^{26} \mathrm{Si},{ }^{30} \mathrm{~S}$, and ${ }^{34} \mathrm{Ar}$ in Ref. [6]. Full details of the required formalism and the methodology used are presented here. A preliminary discussion of the partial cross-section parallel momentum distributions of the reaction residues to be expected following two nucleon removal is also discussed elsewhere [11].

The generalization of the formalism of Ref. [5] to include the one-nucleon stripping plus one-nucleon diffraction events is presented in Sec. II. The inclusion of isospin dependence is discussed in Sec. IIF. The methodology and partial crosssection calculations for the nuclei ${ }^{28} \mathrm{Mg},{ }^{26} \mathrm{Si},{ }^{30} \mathrm{~S},{ }^{34} \mathrm{Ar}$, and ${ }^{54} \mathrm{Ti}$ are presented in Sec. III and the resulting cross sections are discussed in Sec. IV. Section V contains a short summary and concluding remarks.

The application of the present ideas and methodology to reactions involving the removal of two like-nucleons of the excess species, such as for two-neutron removal from neutron-rich systems or of two-proton removal from nuclei near the proton dripline, requires additional consideration of contributions to the cross section from indirect (two-step) mechanisms.

\section{FORMALISM}

We discuss two-nucleon knockout from a secondary projectile beam at intermediate energies of order $100 \mathrm{MeV}$ per nucleon. We consider the projectile to be an antisymmetrized $A+2$ nucleon system with many-body wave function $\Psi(A, 1,2)$. We do not enumerate explicitly all nucleon coordinates. Here, $\Psi(A, 1,2)$ is interpreted as the shell-model $A+2$ nucleon ground state of the projectiles. This is assumed to carry total angular momentum and isospin $J_{i}$ and $T_{i}$ with projections $M_{i}$ and $\tau_{i}$. Following the sudden removal of two-nucleons in a peripheral, high-speed collision with the target nucleus, the $A$-body residue (or core nucleus) is, in general, found in one of a number of final states $\Phi_{J_{f} M_{f}}(A)$, with spin and isospin $J_{f}, T_{f}$ and projections $M_{f}, \tau_{f}$. The isospin and angular momentum couplings involved are summarized in Fig. 1.

\section{A. Two-nucleon amplitudes}

The two removed nucleons, denoted 1 and 2, are assumed to be removed from a set of one or more active (partially occupied) shell-model single-particle orbitals $\phi_{j}$, having spherical quantum numbers $n(\ell s) j, m$. The removed nucleons are assumed to couple to states with intermediate total angular momentum $I, \mu$ and total isospin $T, \tau$.

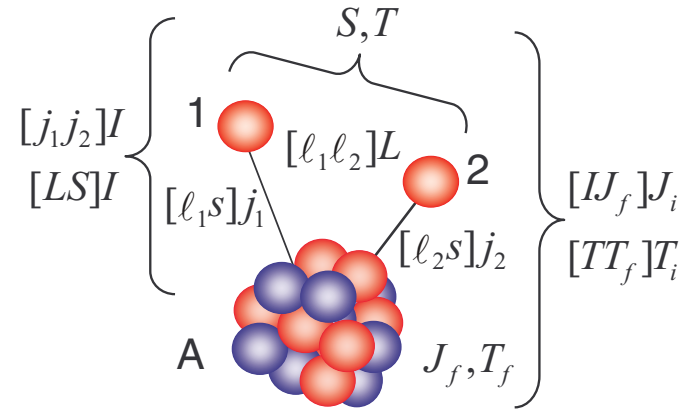

FIG. 1. (Color online) Schematic of the angular momentum couplings used in the description of the two-nucleon knockout reaction.

The shell-model two-nucleon overlap functions for two nucleons in the projectile ground state, relative to a specified residue or core state $f$, is, in general, a sum over several contributing two particle configurations

$$
\begin{aligned}
\Psi_{J_{i} M_{i}}^{(f)}(1,2) & \equiv\left\langle\Phi_{J_{f} M_{f}}(A) \mid \Psi_{J_{i} M_{i}}(A, 1,2)\right\rangle \\
& =\sum_{I \mu \alpha} C_{\alpha}^{J_{i} J_{f} I}\left(I \mu J_{f} M_{f} \mid J_{i} M_{i}\right)\left[\overline{\phi_{j_{1}}(1) \otimes \phi_{j_{2}}(2)}\right]_{I \mu},
\end{aligned}
$$

where $\alpha \equiv\left\{n_{1} \ell_{1} j_{1}, n_{2} \ell_{2} j_{2}\right\}$ denotes each pair of contributing orbitals. The $C_{\alpha}^{J_{i} J_{f} I}$ are the signed two-nucleon amplitudes (TNA) that carry the structure calculation details; in particular, the information on the parentage and phase of each participating two-nucleon configuration. The

$$
\begin{aligned}
{\left[\overline{\phi_{j_{1}}(1) \otimes \phi_{j_{2}}(2)}\right]_{I \mu} } & =-N_{12}\left\langle 1,2\left|\left[a_{j_{1}}^{\dagger} \otimes a_{j_{2}}^{\dagger}\right]_{I \mu}\right| 0\right\rangle \\
& =D_{\alpha} \sum_{m_{1} m_{2}}\left(j_{1} m_{1} j_{2} m_{2} \mid I \mu\right) \\
& \times\left[\phi_{j_{1}}^{m_{1}}(1) \phi_{j_{2}}^{m_{2}}(2)-\phi_{j_{1}}^{m_{1}}(2) \phi_{j_{2}}^{m_{2}}(1)\right]
\end{aligned}
$$

are the normalized, antisymmetrized two-nucleon wave functions with $D_{\alpha}=N_{12} / \sqrt{2}=1 / \sqrt{2\left(1+\delta_{12}\right)}$. So as not to complicate the notation unduely, we do not show the isospin labels and coupling explicitly. We discuss and include these into the formalism in Sec. IIF.

\section{B. Eikonal model and two-nucleon removal mechanisms}

In the eikonal direct reaction theory, the interaction of the two nucleons $(1,2)$ and the $A$-body residue $f$ with the target are described by their elastic $\mathrm{S}$ matrices, $\mathcal{S}_{i}$, see, e.g., Ref. [12]. These $\mathcal{S}_{i}$ are functions of their individual impact parameters $b_{i}$ and we assume they are spin independent. The two removed nucleons can interact either inelastically with the target, and be absorbed from the elastic channel, reflected by an absorption probability $\left(1-\left|\mathcal{S}_{i}\right|^{2}\right)$, or interact elastically with the target, described by $\left|\mathcal{S}_{i}\right|^{2}$.

The total absorption cross section of the reaction is the projectile ground-state expectation value

$$
\sigma_{\mathrm{abs}}=\frac{1}{2 J_{i}+1} \sum_{M_{i}} \int d \boldsymbol{b}\left\langle\Psi_{J_{i} M_{i}}\left|\left[1-\left|\mathcal{S}_{f} \mathcal{S}_{1} \mathcal{S}_{2}\right|^{2}\right]\right| \Psi_{J_{i} M_{i}}\right\rangle,
$$


integrated over all values of the center-of-mass (c.m.) impact parameter, $b$, of the projectile. Expanding $1=\prod_{i}\{[1-$ $\left.\left.\left|\mathcal{S}_{i}\right|^{2}\right]+\left|\mathcal{S}_{i}\right|^{2}\right\}$, and retaining only terms involving $\left|\mathcal{S}_{f}\right|^{2}$, the remaining contributions to the absorption cross section involve the operators

$$
\begin{aligned}
\mathcal{O}= & \left|\mathcal{S}_{f}\right|^{2}\left[\left(1-\left|\mathcal{S}_{1}\right|^{2}\right)\left(1-\left|\mathcal{S}_{2}\right|^{2}\right)+\left|\mathcal{S}_{1}\right|^{2}\left(1-\left|\mathcal{S}_{2}\right|^{2}\right)\right. \\
& \left.+\left|\mathcal{S}_{2}\right|^{2}\left(1-\left|\mathcal{S}_{1}\right|^{2}\right)\right]
\end{aligned}
$$

These terms reflect the underlying two-nucleon removal reaction mechanism in which the residue interacts at most elastically with the target and is measured in the final state. For such processes we need consider, and have retained, only terms in the total absorption cross section that contain the core transmission or survival factor, $\left|\mathcal{S}_{f}\right|^{2}$.

In Ref. [5], the stripping (inelastic breakup) contribution to the two-nucleon removal cross section was formulated and analyzed. This cross section results from the $\left(1-\left|\mathcal{S}_{1}\right|^{2}\right)(1-$ $\left|\mathcal{S}_{2}\right|^{2}$ ) term above, i.e.,

$$
\begin{aligned}
\sigma_{\text {str }}= & \frac{1}{2 J_{i}+1} \sum_{M_{i}} \int d \boldsymbol{b}\left\langle\left.\Psi_{J_{i} M_{i}}|| \mathcal{S}_{f}\right|^{2}\left(1-\left|\mathcal{S}_{1}\right|^{2}\right)\right. \\
& \left(1-\left|\mathcal{S}_{2}\right|^{2}\right)\left|\Psi_{J_{i} M_{i}}\right\rangle .
\end{aligned}
$$

There are also contributions to the knockout cross section from the remaining terms in Eq. (4), describing events where one of the two nucleons, say nucleon 1, is removed in an elastic collision with the target and so emerges from the collision, described by $\left|\mathcal{S}_{1}\right|^{2}$, whereas the second nucleon, 2, is absorbed. These contributions thus take the form

$\sigma_{1}=\frac{1}{2 J_{i}+1} \sum_{M_{i}} \int d \boldsymbol{b}\left\langle\left.\Psi_{J_{i} M_{i}}|| \mathcal{S}_{f}\right|^{2}\left|\mathcal{S}_{1}\right|^{2}\left(1-\left|\mathcal{S}_{2}\right|^{2}\right) \mid \Psi_{J_{i} M_{i}}\right\rangle$.

We make the simplifying approximation that the residuetarget $S$ matrix is diagonal with respect to different final states $f$ of the residue and that this diagonal interaction is the same as that for the residue ground state (denoted $\mathcal{S}_{c}$ ) for all final states $f$. This has been termed the spectator-core approximation when used in single-nucleon knockout [13]. It assumes that amplitudes for dynamical excitation of the core during the collision are small. We also neglect explicit recoil effects associated with the heavy, mass $A$ residue. Thus, we replace

$$
\left\langle\left.\Phi_{J_{f}^{\prime} M_{f}^{\prime}}(A)|| \mathcal{S}_{f}\right|^{2} \mid \Phi_{J_{f} M_{f}}(A)\right\rangle=\left|\mathcal{S}_{c}(b)\right|^{2} \delta_{f f^{\prime}} \delta_{J_{f}} J_{f}^{\prime} \delta_{M_{f} M_{f}^{\prime}},
$$

where $b$ is the projectile c.m. impact parameter.

Having made the spectator-core and the no-recoil approximations, the inclusive stripping cross section is the sum of the incoherent contributions due to each residue final state. So, with $\hat{J}^{2}=(2 J+1)$, then

$$
\begin{aligned}
\sigma_{\text {str }} \sum_{f} \sigma_{s t r}^{(f)}= & \sum_{f}\left[\int d \boldsymbol{b}\left|\mathcal{S}_{c}\right|^{2} \frac{1}{\hat{J}_{i}^{2}}\right. \\
& \left.\times \sum_{M_{i}}\left\langle\Psi_{J_{i} M_{i}}^{(f)}\left|\left(1-\left|\mathcal{S}_{1}\right|^{2}\right)\left(1-\left|\mathcal{S}_{2}\right|^{2}\right)\right| \Psi_{J_{i} M_{i}}^{(f)}\right\rangle\right] .
\end{aligned}
$$

The analogous stripping-diffraction contributions, of major interest here, involve $\sigma_{1}+\sigma_{2}$, where, for diffraction of nucleon 1

$$
\begin{aligned}
\sigma_{1} & =\sum_{f} \sigma_{1}^{(f)} \\
& =\sum_{f}\left[\int d \boldsymbol{b}\left|\mathcal{S}_{c}\right|^{2} \frac{1}{\hat{J}_{i}^{2}} \sum_{M_{i}}\left\langle\left.\left.\Psi_{J_{i} M_{i}}^{(f)}|| \mathcal{S}_{1}\right|^{2}\left(1-\mid \mathcal{S}_{2}\right)\right|^{2} \mid \Psi_{J_{i} M_{i}}^{(f)}\right\rangle\right],
\end{aligned}
$$

and similarly for $\sigma_{2}$ and the diffraction of nucleon 2 .

Common to all of these terms, the bra-ket denotes integration over the spatial coordinates of the two removed nucleons, $\boldsymbol{r}_{1}$ and $\boldsymbol{r}_{2}$, and the integration over all spin variables, denoted here, following Ref. [5], by

$$
\left\langle\Psi_{J_{i} M_{i}}^{(f)}|\ldots| \Psi_{J_{i} M_{i}}^{(f)}\right\rangle=\int d \boldsymbol{r}_{1} \int d \boldsymbol{r}_{2}\left\langle\Psi_{J_{i} M_{i}}^{(f)}|\ldots| \Psi_{J_{i} M_{i}}^{(f)}\right\rangle_{\mathrm{sp}}
$$

Because the $S$ matrices with the target are assumed spin independent, we require only the spin-average of the twonucleon wave functions,

$$
\begin{aligned}
\frac{1}{\hat{J}_{i}^{2}} & \sum_{M_{i}}\left\langle\Psi_{J_{i} M_{i}}^{(f)} \mid \Psi_{J_{i} M_{i}}^{(f)}\right\rangle_{\mathrm{sp}} \\
= & \frac{1}{\hat{J}_{i}^{2}} \sum_{M_{f} M_{i} I \mu \alpha I^{\prime} \mu^{\prime} \alpha^{\prime}} C_{\alpha^{\prime}}^{J_{i} J_{f} I^{\prime}} C_{\alpha}^{J_{i} J_{f} I} \\
& \times\left(I^{\prime} \mu^{\prime} J_{f} M_{f} \mid J_{i} M_{i}\right)\left(I \mu J_{f} M_{f} \mid J_{i} M_{i}\right) \\
& \times\left\langle\left[\overline{\phi_{j_{1}^{\prime}}(1) \otimes \phi_{j_{2}^{\prime}}(2)}\right]_{I^{\prime} \mu^{\prime}} \mid\left[\overline{\phi_{j_{1}}(1) \otimes \phi_{j_{2}}(2)}\right]_{I \mu}\right\rangle_{\mathrm{sp}} .
\end{aligned}
$$

Upon using Eqs. (1) and (2) this reduces to

$$
\begin{aligned}
\frac{1}{\hat{J}_{i}^{2}} \sum_{M_{i}}\left\langle\Psi_{J_{i} M_{i}}^{(f)} \mid \Psi_{J_{i} M_{i}}^{(f)}\right\rangle_{\mathrm{sp}} \\
=\sum_{I \alpha \alpha^{\prime}} 2 D_{\alpha} D_{\alpha^{\prime}} \frac{C_{\alpha^{\prime}}^{J_{i} J_{f} I} C_{\alpha}^{J_{i} J_{f} I}}{\hat{I}^{2}} \\
\quad \times \sum_{m_{1} m_{2} m_{1}^{\prime} m_{2}^{\prime} \mu}\left(j_{1} m_{1} j_{2} m_{2} \mid I \mu\right)\left(j_{1}^{\prime} m_{1}^{\prime} j_{2}^{\prime} m_{2}^{\prime} \mid I \mu\right) \\
\quad \times\left[\left(\phi_{j_{1}^{\prime}}^{m_{1}^{\prime}} \mid \phi_{j_{1}}^{m_{1}}\right)_{\mathrm{sp}}\left(\phi_{j_{2}^{\prime}}^{m_{2}^{\prime}} \mid \phi_{j_{2}}^{m_{2}}\right)_{\mathrm{sp}}-\left(\phi_{j_{1}^{\prime}}^{m_{1}^{\prime}} \mid \phi_{j_{2}}^{m_{2}}\right)_{\mathrm{sp}}\left(\phi_{j_{2}^{\prime}}^{m_{2}^{\prime}} \mid \phi_{j_{1}}^{m_{1}}\right)_{\mathrm{sp}}\right]
\end{aligned}
$$

in which we have assumed that we will need to compute only matrix elements of operators that are symmetric with respect to the nucleon labels 1 and 2 . We have also introduced the alternative bra-kets (..|..), which are used to denote integrations over the coordinates of a single nucleon. We refer to terms due to the first product in the final bracket as being direct and terms due to the second product as exchange terms. The spin-averages for each single-particle state, with the nucleon 
spin $s=1 / 2$ understood, yields the multipole expansion [14]

$$
\begin{aligned}
\left(\phi_{j^{\prime}}^{m^{\prime}} \mid \phi_{j}^{m}\right)_{\mathrm{sp}}= & \sum_{k q}\left(j^{\prime} m^{\prime} k q \mid j m\right)\left[\frac{\hat{\ell} \hat{\ell}^{\prime} \hat{j}^{\prime}}{\sqrt{4 \pi}}(-1)^{2 s+j+j^{\prime}-\ell}\left(\ell 0 \ell^{\prime} 0 \mid k 0\right)\right. \\
& \left.\times W\left(j s k \ell^{\prime} ; \ell j^{\prime}\right) u_{j^{\prime} \ell^{\prime}}(r) u_{j \ell}(r) Y_{k q}(\hat{\boldsymbol{r}})\right] \\
\equiv & \sum_{k q}\left(j^{\prime} m^{\prime} k q \mid j m\right)\left\langle\left\langle j^{\prime} \ell^{\prime}\left|\mathcal{O}_{k q}(\boldsymbol{r})\right| j \ell\right\rangle\right\rangle,
\end{aligned}
$$

where the $u_{j \ell}(r)$ are the single-particle radial wave functions and $\langle\langle\ldots\rangle\rangle$ has been used as shorthand for the entire squarebracketed expression.

These single-particle spin averages now enter the knockout calculations together with a corresponding nucleonic operator, e.g., $\left|\mathcal{S}_{i}\right|^{2}$ or $\left(1-\left|\mathcal{S}_{i}\right|^{2}\right)$, and are integrated over the appropriate single-particle position coordinate $\boldsymbol{r}_{i}$. For the one-nucleon absorption (stripping) operator we write [5]

$$
\begin{aligned}
\left(\phi_{j^{\prime}}^{m^{\prime}}\left|\left(1-|\mathcal{S}|^{2}\right)\right| \phi_{j}^{m}\right)= & \sum_{k q}\left(j^{\prime} m^{\prime} k q \mid j m\right) \int d \boldsymbol{r}\left(1-|\mathcal{S}|^{2}\right) \\
& \times\left\langle\left\langle j^{\prime} \ell^{\prime}\left|\mathcal{O}_{k q}(\boldsymbol{r})\right| j \ell\right\rangle\right\rangle \\
\equiv & \sum_{k q}\left(j^{\prime} m^{\prime} k q \mid j m\right)\left\{j^{\prime} \ell^{\prime}\left|\mathcal{F}_{k q}(b)\right| j \ell\right\},
\end{aligned}
$$

which defines the bracketed quantities $\left\{j^{\prime} \ell^{\prime}\left|\mathcal{F}_{k q}(b)\right| j \ell\right\}$, functions of the single-particle angular momenta indicated and the projectile's c.m. impact parameter $b$. Analogous expressions will appear when we deal with the other operator forms arising from the stripping-diffraction removal mechanism.

These stripping-diffraction terms, $\sigma_{i}^{(f)}$, require further attention. It should be noted that the cross sections, as presently shown in Eq. (9), include all processes in which nucleon $i$ survives the collision, including those in which it remains bound to the residue. These processes correspond to a single-nucleon absorption from the projectile, populating bound final states of an $(A+1)$-body residue. Such terms could be ignored in an earlier discussion [15] of the nuclear breakup of Borromean nuclei, such as ${ }^{11} \mathrm{Li}$, where it can be assumed that there are no $A+1$-body bound final states of the residue (core) and the (nonabsorbed) valence neutron.

To isolate the contributions that dissociate nucleon $i$ from the residue, we use closure and the complete set of states of the nucleon-core system. So, we avoid explicit reference to the continuum of nucleon-residue unbound states by rewriting Eq. (9) as

$$
\left|\mathcal{S}_{i}\right|^{2} \rightarrow \mathcal{S}_{i}^{*}\left[1-\sum_{j^{\prime \prime} m^{\prime \prime}} \mid \phi_{j^{\prime \prime}}^{m^{\prime \prime}}\right)\left(\phi_{j^{\prime \prime}}^{m^{\prime \prime}} \mid\right] \mathcal{S}_{i},
$$

where the notation implies a summation over (projection off) bound eigenstates $n\left(\ell^{\prime \prime} s\right) j^{\prime \prime}, m^{\prime \prime}$ of the nucleon $i$ and the core. Here we include all active single-particle orbitals in this sum. It follows therefore that the analog of Eq. (14), from those parts of the $\left|\mathcal{S}_{i}\right|^{2}$ terms that result in two-nucleon removal are more complex. Using Eq. (15), then we must replace

$$
\begin{gathered}
\left(\left.\phi_{j^{\prime}}^{m^{\prime}}|| \mathcal{S}\right|^{2} \mid \phi_{j}^{m}\right) \rightarrow\left(\left.\phi_{j^{\prime}}^{m^{\prime}}|| \mathcal{S}\right|^{2} \mid \phi_{j}^{m}\right)-\sum_{j^{\prime \prime} m^{\prime \prime}}\left(\phi_{j^{\prime \prime}}^{m^{\prime \prime}}|\mathcal{S}| \phi_{j}^{m}\right)\left(\phi_{j^{\prime \prime}}^{m^{\prime \prime}}|\mathcal{S}| \phi_{j^{\prime}}^{m^{\prime}}\right)^{*} \\
=\sum_{k q}\left(j^{\prime} m^{\prime} k q \mid j m\right)\left\{j^{\prime} \ell^{\prime}\left|\mathcal{H}_{k q}(b)\right| j \ell\right\} \\
\quad-\sum_{j^{\prime \prime} m^{\prime \prime}}\left[\sum_{k^{\prime} q^{\prime} k^{\prime \prime} q^{\prime \prime}}\left(j^{\prime \prime} m^{\prime \prime} k^{\prime} q^{\prime} \mid j m\right)\left(j^{\prime \prime} m^{\prime \prime} k^{\prime \prime} q^{\prime \prime} \mid j^{\prime} m^{\prime}\right)\right. \\
\left.\times\left\{j^{\prime \prime} \ell^{\prime \prime}\left|\mathcal{I}_{k^{\prime} q^{\prime}}(b)\right| j \ell\right\}\left\{j^{\prime \prime} \ell^{\prime \prime}\left|\mathcal{I}_{k^{\prime \prime}} q^{\prime \prime}(b)\right| j^{\prime} \ell^{\prime}\right\}^{*}\right],
\end{gathered}
$$

where we have introduced, by analogy with Eq. (14),

$$
\begin{aligned}
\left(\left.\phi_{j^{\prime}}^{m^{\prime}}|| \mathcal{S}\right|^{2} \mid \phi_{j}^{m}\right)= & \sum_{k q}\left(j^{\prime} m^{\prime} k q \mid j m\right) \\
& \times \int d \boldsymbol{r}|\mathcal{S}|^{2}\left\langle\left\langle j^{\prime} \ell^{\prime}\left|\mathcal{O}_{k q}(\boldsymbol{r})\right| j \ell\right\rangle\right\rangle \\
\equiv & \sum_{k q}\left(j^{\prime} m^{\prime} k q \mid j m\right)\left\{j^{\prime} \ell^{\prime}\left|\mathcal{H}_{k q}(b)\right| j \ell\right\} \\
\left(\phi_{j^{\prime}}^{m^{\prime}}|\mathcal{S}| \phi_{j}^{m}\right)= & \sum_{k q}\left(j^{\prime} m^{\prime} k q \mid j m\right) \int d \boldsymbol{r} \mathcal{S}\left\langle\left\langle j^{\prime} \ell^{\prime}\left|\mathcal{O}_{k q}(\boldsymbol{r})\right| j \ell\right\rangle\right\rangle \\
\equiv & \sum_{k q}\left(j^{\prime} m^{\prime} k q \mid j m\right)\left\{j^{\prime} \ell^{\prime}\left|\mathcal{I}_{k q}(b)\right| j \ell\right\}
\end{aligned}
$$

\section{Two-nucleon stripping terms}

Complete expressions, following the evaluation of the stripping operator terms,

$$
\mathcal{K}_{1}(1,2)=\left(1-\left|\mathcal{S}_{1}\right|^{2}\right)\left(1-\left|\mathcal{S}_{2}\right|^{2}\right)
$$

were derived in Ref. [5]. These are

$$
\begin{aligned}
\frac{1}{\hat{J}_{i}^{2}} \sum_{M_{i}}\left\langle\Psi_{J_{i} M_{i}}^{(f)}\left|\mathcal{K}_{1}(1,2)\right| \Psi_{J_{i} M_{i}}^{(f)}\right\rangle= & \sum_{\alpha \alpha^{\prime} I} 2 D_{\alpha} D_{\alpha^{\prime}} C_{\alpha^{\prime}}^{J_{J^{\prime}} J_{f} I} C_{\alpha}^{J_{i} J_{f} I} \hat{j}_{1} \hat{j}_{2} \\
& \times \sum_{K Q} \frac{(-)^{Q}}{\hat{K}^{2}} \\
& \times[\text { direct }- \text { exchange], (20) }
\end{aligned}
$$

where

$$
\begin{aligned}
\text { direct } \equiv & (-)^{I-j_{1}-j_{2}^{\prime}} W\left(j_{1} j_{1}^{\prime} j_{2} j_{2}^{\prime} ; K I\right)\left\{j_{1}^{\prime} \ell_{1}^{\prime}\left|\mathcal{F}_{K-Q}(b)\right| j_{1} \ell_{1}\right\} \\
& \times\left\{j_{2}^{\prime} \ell_{2}^{\prime}\left|\mathcal{F}_{K Q}(b)\right| j_{2} \ell_{2}\right\}, \\
\text { exchange } \equiv & (-)^{j_{2}^{\prime}-j_{1}} W\left(j_{2} j_{1}^{\prime} j_{1} j_{2}^{\prime} ; K I\right)\left\{j_{1}^{\prime} \ell_{1}^{\prime}\left|\mathcal{F}_{K-Q}(b)\right| j_{2} \ell_{2}\right\} \\
& \times\left\{j_{2}^{\prime} \ell_{2}^{\prime}\left|\mathcal{F}_{K Q}(b)\right| j_{1} \ell_{1}\right\} .
\end{aligned}
$$

\section{Nucleon stripping and diffraction terms}

The new mechanisms discussed here are the strippingdiffraction terms. The contributions from the first terms on the right-hand side of Eq. (16) are structurally similar to the stripping terms. Considering the (symmetric) combination of 
operators

$$
\mathcal{K}_{2}(1,2)=\left|\mathcal{S}_{1}\right|^{2}\left(1-\left|\mathcal{S}_{2}\right|^{2}\right)+\left(1-\left|\mathcal{S}_{1}\right|^{2}\right)\left|\mathcal{S}_{2}\right|^{2},
$$

then one finds

$$
\begin{aligned}
\frac{1}{\hat{J}_{i}^{2}} \sum_{M_{i}}\left\langle\Psi_{J_{i} M_{i}}^{(f)}\left|\mathcal{K}_{2}(1,2)\right| \Psi_{J_{i} M_{i}}^{(f)}\right\rangle= & \sum_{\alpha \alpha^{\prime} I} 2 D_{\alpha} D_{\alpha^{\prime}} C_{\alpha^{\prime}}^{J_{i} J_{f} I} C_{\alpha}^{J_{i} J_{f} I} \hat{j}_{1} \hat{j}_{2} \\
& \times \sum_{K Q} \frac{(-)^{Q}}{\hat{K}^{2}} \\
& \times\left[\text { direct }^{\prime}-\text { exchange' }\right],
\end{aligned}
$$

where now

direct $^{\prime}$

$$
\begin{aligned}
\equiv & (-)^{I-j_{1}-j_{2}^{\prime}} W\left(j_{1} j_{1}^{\prime} j_{2} j_{2}^{\prime} ; K I\right) \\
& \times\left[\left\{j_{1}^{\prime} \ell_{1}^{\prime}\left|\mathcal{H}_{K-Q}(b)\right| j_{1} \ell_{1}\right\}\left\{j_{2}^{\prime} \ell_{2}^{\prime}\left|\mathcal{F}_{K Q}(b)\right| j_{2} \ell_{2}\right\}\right. \\
& \left.+\left\{j_{1}^{\prime} \ell_{1}^{\prime}\left|\mathcal{F}_{K Q}(b)\right| j_{1} \ell_{1}\right\}\left\{j_{2}^{\prime} \ell_{2}^{\prime}\left|\mathcal{H}_{K-Q}(b)\right| j_{2} \ell_{2}\right\}\right]
\end{aligned}
$$

exchange'

$$
\begin{aligned}
\equiv & (-)^{j_{2}^{\prime}-j_{1}} W\left(j_{2} j_{1}^{\prime} j_{1} j_{2}^{\prime} ; K I\right)\left[\left\{j_{1}^{\prime} \ell_{1}^{\prime}\left|\mathcal{H}_{K-Q}(b)\right| j_{2} \ell_{2}\right\}\right. \\
& \times\left\{j_{2}^{\prime} \ell_{2}^{\prime}\left|\mathcal{F}_{K Q}(b)\right| j_{1} \ell_{1}\right\}+\left\{j_{1}^{\prime} \ell_{1}^{\prime}\left|\mathcal{F}_{K Q}(b)\right| j_{2} \ell_{2}\right\} \\
& \left.\times\left\{j_{2}^{\prime} \ell_{2}^{\prime}\left|\mathcal{H}_{K-Q}(b)\right| j_{1} \ell_{1}\right\}\right] .
\end{aligned}
$$

This clearly includes the contributions from both $\sigma_{1}^{(f)}$ and $\sigma_{2}^{(f)}$. The more complicated contributions, from the second (excluded bound states) terms on the right-hand side of Eq. (16), now involve the symmetric operator

$$
\begin{aligned}
\mathcal{K}_{3}(1,2)= & \sum_{j^{\prime \prime} m^{\prime \prime}}\left[\mathcal{S}_{1}^{*} \mid \phi_{j^{\prime \prime}}^{m^{\prime \prime}}\right)\left(\phi_{j^{\prime \prime}}^{m^{\prime \prime}} \mid \mathcal{S}_{1}\left(1-\left|\mathcal{S}_{2}\right|^{2}\right)\right. \\
& \left.+\left(1-\left|\mathcal{S}_{1}\right|^{2}\right) \mathcal{S}_{2}^{*} \mid \phi_{j^{\prime \prime}}^{m^{\prime \prime}}\right)\left(\phi_{j^{\prime \prime}}^{m^{\prime \prime}} \mid \mathcal{S}_{2}\right]
\end{aligned}
$$

giving

$$
\begin{aligned}
\frac{1}{\hat{J}_{i}^{2}} & \sum_{M_{i}}\left\langle\Psi_{J_{i} M_{i}}^{(f)}\left|\mathcal{K}_{3}(1,2)\right| \Psi_{J_{i} M_{i}}^{(f)}\right\rangle=\sum_{\alpha \alpha^{\prime} I} 2 D_{\alpha} D_{\alpha^{\prime}} C_{\alpha^{\prime}}^{J_{i} J_{f} I} C_{\alpha}^{J_{i} J_{f} I} \hat{j}_{1} \hat{j}_{2} \\
& \times \sum_{K Q k^{\prime} q^{\prime} k^{\prime \prime} q^{\prime \prime}} \frac{(-)^{k^{\prime \prime}-k^{\prime}+Q}}{\hat{k}^{\prime}}\left(k^{\prime \prime} q^{\prime \prime} K-Q \mid k^{\prime} q^{\prime}\right) \\
& \times \sum_{j^{\prime \prime}}\left[\operatorname{direct}^{\prime \prime}-\text { exchange } \mathrm{e}^{\prime \prime}\right]
\end{aligned}
$$

where now

$$
\begin{aligned}
& \text { direct }^{\prime \prime} \\
& \equiv W\left(j_{1} j_{1}^{\prime} j_{2} j_{2}^{\prime} ; K I\right)\left[(-)^{I-j_{1}^{\prime}-j_{2}^{\prime}} \hat{j}_{1}^{\prime} W\left(j^{\prime \prime} j_{1}^{\prime} k^{\prime} K ; k^{\prime \prime} j_{1}\right)\right. \\
& \times\left\{j^{\prime \prime} \ell^{\prime \prime}\left|\mathcal{I}_{k^{\prime} q^{\prime}}(b)\right| j_{1} \ell_{1}\right\}\left\{j^{\prime \prime} \ell^{\prime \prime}\left|\mathcal{I}_{k^{\prime \prime} q^{\prime \prime}}(b)\right| j_{1}^{\prime} \ell_{1}^{\prime}\right\}^{*} \\
& \times\left\{j_{2}^{\prime} \ell_{2}^{\prime}\left|\mathcal{F}_{K Q}(b)\right| j_{2} \ell_{2}\right\}+(-)^{I-j_{1}-j_{2}} \hat{j}_{2}^{\prime} \\
& \times W\left(j^{\prime \prime} j_{2}^{\prime} k^{\prime} K ; k^{\prime \prime} j_{2}\right)\left\{j^{\prime \prime} \ell^{\prime \prime}\left|\mathcal{I}_{k^{\prime} q^{\prime}}(b)\right| j_{2} \ell_{2}\right\} \\
&\left.\times\left\{j^{\prime \prime} \ell^{\prime \prime}\left|\mathcal{I}_{k^{\prime \prime} q^{\prime \prime}}(b)\right| j_{2}^{\prime} \ell_{2}^{\prime}\right\}^{*}\left\{j_{1}^{\prime} \ell_{1}^{\prime}\left|\mathcal{F}_{K Q}(b)\right| j_{1} \ell_{1}\right\}\right] \\
& \operatorname{exchange^{\prime \prime }} \\
& \equiv W\left(j_{2} j_{1}^{\prime} j_{1} j_{2}^{\prime} ; K I\right)\left[(-)^{j_{1}-j_{1}^{\prime}+j_{2}-j_{2}^{\prime}} \hat{j}_{1}^{\prime} W\left(j^{\prime \prime} j_{1}^{\prime} k^{\prime} K ; k^{\prime \prime} j_{2}\right)\right. \\
& \times\left\{j^{\prime \prime} \ell^{\prime \prime}\left|\mathcal{I}_{k^{\prime} q^{\prime}}(b)\right| j_{2} \ell_{2}\right\}\left\{j^{\prime \prime} \ell^{\prime \prime}\left|\mathcal{I}_{k^{\prime \prime} q^{\prime \prime}}(b)\right| j_{1}^{\prime} \ell_{1}^{\prime}\right\}^{*} \\
& \times\left\{j_{2}^{\prime} \ell_{2}^{\prime}\left|\mathcal{F}_{K Q}(b)\right| j_{1} \ell_{1}\right\}+\hat{j}_{2}^{\prime} W\left(j^{\prime \prime} j_{2}^{\prime} k^{\prime} K ; k^{\prime \prime} j_{1}\right) \\
& \times\left\{j^{\prime \prime} \ell^{\prime \prime}\left|\mathcal{I}_{k^{\prime} q^{\prime}}(b)\right| j_{1} \ell_{1}\right\}\left\{j^{\prime \prime} \ell^{\prime \prime}\left|\mathcal{I}_{k^{\prime \prime} q^{\prime \prime}}(b)\right| j_{2}^{\prime} \ell_{2}^{\prime}\right\}^{*} \\
&\left.\times\left\{j_{1}^{\prime} \ell_{1}^{\prime}\left|\mathcal{F}_{K Q}(b)\right| j_{2} \ell_{2}\right\}\right]
\end{aligned}
$$

Subtracting Eq. (28) from Eq. (24) now yields the strippingdiffraction contributions from both Eq. (9) and its analog for $\sigma_{2}^{(f)}$. We denote these contributions as $\sigma_{\text {str-diff }}^{(f)}=\sigma_{\text {diff, } 1}^{(f)}+$ $\sigma_{\text {diff, }, 2}^{(f)}$, where of course $\sigma_{\text {diff, } 1}^{(f)}=\sigma_{\text {diff }, 2}^{(f)}$.

\section{E. Two-nucleon diffraction estimate}

Finally, in the following we include an estimate of the (smaller) cross section due to the removal of both (tightly bound) nucleons by elastic dissociation. Our estimate makes use of the reduction in the cross section when each nucleon is elastically dissociated compared to it being stripped, that is $\sigma_{\text {diff,i }}^{(f)} / \sigma_{\text {str }}^{(f)}$. We thus estimate the two-nucleon elastic breakup cross section to be $\sigma_{\text {diff }}^{(f)} \approx\left[\sigma_{\text {diff, } \mathrm{i}}^{(f)} / \sigma_{\text {str }}^{(f)}\right]^{2} \sigma_{\text {str }}^{(f)}$. We will see in the following section that, typically, $\sigma_{\mathrm{diff}, \mathrm{i}}^{(f)} / \sigma_{\text {str }}^{(f)} \approx 0.35-0.4$ for the cases considered here, hence $\sigma_{\text {diff }}^{(f)}$ will make a contribution of only $6 \%-8 \%$ to the total two-nucleon removal partial cross sections.

\section{F. Isospin dependence}

The inclusion of isospin labels in Eq. (1) and the subsequent equations leads to rather simple modifications. Eq. (1) becomes

$$
\begin{aligned}
\Psi_{J_{i} M_{i} T_{i} \tau_{i}}^{(f)}(1,2) \equiv & \left\langle\Phi_{J_{f} M_{f} T_{f} \tau_{f}}(A) \mid \Psi_{J_{i} M_{i} T_{i} \tau_{i}}(A, 1,2)\right\rangle \\
= & \sum_{I \mu \alpha T \tau} C_{\alpha}^{J_{i} J_{f} I T_{i} T_{f} T}\left(I \mu J_{f} M_{f} \mid J_{i} M_{i}\right) \\
& \times\left(T \tau T_{f} \tau_{f} \mid T_{i} \tau_{i}\right)\left[\overline{\phi_{j_{1}}(1) \otimes \phi_{j_{2}}(2)}\right]_{I \mu}^{T \tau}
\end{aligned}
$$

where now

$$
\begin{aligned}
& {\left[\overline{\phi_{j_{1}}(1) \otimes \phi_{j_{2}}(2)}\right]_{I \mu}^{T \tau}} \\
& =D_{\alpha} \sum_{m_{1} m_{2}}\left(j_{1} m_{1} j_{2} m_{2} \mid I \mu\right) \\
& \times\left[\phi_{j_{1}}^{m_{1}}(1) \phi_{j_{2}}^{m_{2}}(2)-(-)^{1+T} \phi_{j_{1}}^{m_{1}}(2) \phi_{j_{2}}^{m_{2}}(1)\right] \chi_{T \tau}(1,2) .
\end{aligned}
$$

Thus, isospin introduces an additional phase factor of $(-)^{1+T}$ in front of the exchange terms in Eqs. (20) through (30). The two-nucleon amplitudes $C$ are also $T$ dependent. In addition, the final expressions for the cross sections must be multiplied by the square of the isospin coupling ClebshGordan coefficient $\left(T \tau T_{f} \tau_{f} \mid T_{i} \tau_{i}\right)$.

\section{APPLICATIONS TO TWO-PROTON AND TWO-NEUTRON KNOCKOUT}

The formalism presented here now allows a complete dynamical calculation and a quantitative comparison of the theoretical cross sections with recent measurements. We do so for the two-proton removal reactions from neutron-rich ${ }^{28} \mathrm{Mg}$ [4] and ${ }^{54} \mathrm{Ti}$ [9], at 83.2 and $72 \mathrm{MeV}$ per nucleon, respectively, and for the two-neutron removal reactions from the neutron-deficient $s d$-shell systems ${ }^{26} \mathrm{Si},{ }^{30} \mathrm{~S}$, and ${ }^{34} \mathrm{Ar}$, at 109, 111, and $110 \mathrm{MeV}$ per nucleon [6]. The ground-stateto-ground-state (g.s.) two-nucleon separation energies in all cases are large, because we remove nucleons of the deficient species from the well-bound Fermi surfaces. They are also 
rather similar, being 30.0, 34.0, 34.3, 32.3, and 27.8 MeV for the ${ }^{28} \mathrm{Mg},{ }^{26} \mathrm{Si},{ }^{30} \mathrm{~S},{ }^{34} \mathrm{Ar}$, and ${ }^{54} \mathrm{Ti}$ projectiles, respectively.

\section{A. Reaction and structure methodology}

The reactions are analyzed within a consistent framework. In all cases the two-nucleon amplitudes (TNA) $C_{\alpha}^{J_{i} J_{f} I}$ are given by large-basis shell-model calculations, using the code OXBASH [16]. Our $s d$-shell test case systems, ${ }^{28} \mathrm{Mg},{ }^{26} \mathrm{Si},{ }^{30} \mathrm{~S}$, and ${ }^{34} \mathrm{Ar}$, were calculated using the USD interaction [17] in the full $s d$-shell model space. For ${ }^{54} \mathrm{Ti}$, with two protons in the $p f$ shell, we discuss only the ${ }^{52} \mathrm{Ca}$ ground-state partial cross section, see also Ref. [9]. The TNA in this case were obtained using the GXPF1 Hamiltonian [18] within the full $f p$ shell. The amplitudes in the case of ${ }^{28} \mathrm{Mg}$ were already shown explicitly in Table II of Ref. [5]. A typographical error in one heading of that table should be noted [19].

The nucleon- and residue-target $S$-matrices $\mathcal{S}$ and the nucleon single-particle states $\phi_{j}$ were calculated as outlined in Ref. [20] and are constrained by spherical Hartree-Fock (HF) calculations. The point neutron and proton densities of the mass- $A$ residues were taken from $\mathrm{HF}$ calculations based on the recent Skyrme SkX parametrization [21]. This was determined from a large data set on spherical nuclei, including many nuclei far from stability. The resulting root-mean-squared (rms) matter radii were $3.009,2.915,3.049,3.153$, and $3.632 \mathrm{fm}$ for the ${ }^{26} \mathrm{Ne},{ }^{24} \mathrm{Si},{ }^{28} \mathrm{~S},{ }^{32} \mathrm{Ar}$, and ${ }^{52} \mathrm{Ca}$ residues, respectively. The Gaussian nucleon-nucleon $(N N)$ effective interaction of Ref. [22] was assumed, with a range of $0.5 \mathrm{fm}$, and strength determined, in the usual way, by the free $p p$ and $n p$ cross sections and the real-to-imaginary ratios of the forward $N N$ scattering amplitudes [23]. As in earlier analyses [24], the density of the ${ }^{9} \mathrm{Be}$ target was assumed to be of Gaussian form with rms matter radius of $2.36 \mathrm{fm}$ [25].

The nucleon bound-state radial functions $u_{j \ell}(r)$ were calculated in Woods-Saxon potential wells with a fixed diffuseness parameter of $a=0.7 \mathrm{fm}$. The radius parameters $r_{0}$ of the binding potentials were adjusted, for each $(\ell, j)$ orbital, so that their rms radius was consistent with the HF calculations. This $r_{0}$ adjustment was carried out at the separation energies predicted by the HF calculations. Having determined these $r_{0}$, the bound state wave functions for each transition of interest were then calculated as eigenstates of the Woods-Saxon wells with half the empirical two-nucleon separation energy. A Thomas form spin-orbit potential of strength $6 \mathrm{MeV}$ was also included with the same $\left(r_{0}, a\right)$ geometry parameters. The $s d$-shell cases involved the $1 d_{5 / 2}, 1 d_{3 / 2}$, and $2 s_{1 / 2}$ neutron and proton single-particle orbitals and the ${ }^{54} \mathrm{Ti}$ (g.s.) case the proton $1 f_{7 / 2}, 1 f_{5 / 2}, 2 p_{3 / 2}$, and $2 p_{1 / 2}$ states. All of these active orbitals were included in the projection operator in Eqs. (15) and (17). The phase convention of the $u_{j \ell}(r)$ to be used with the TNA from OXBASH and its two-body interaction library is discussed in Ref. [5].

\section{RESULTS FOR CROSS SECTIONS}

The two-nucleon removal cross sections can now be computed. For each transition, the two-nucleon separation energy used was that of the ground-state transition plus the excitation energy of the final state. To clarify the contributions from the different stripping and diffractive removal mechanisms we first show these separately. Although there is currently no measurement of these individual components, it will become clear that such a measurement would provide an excellent additional test of these reaction mechanisms.

In Tables I and II we show the contributions to the two-nucleon knockout partial cross sections $\sigma^{(f)}$ arising from the stripping, $\sigma_{\text {str }}^{(f)}$, the stripping-diffraction $\sigma_{\text {str-diff }}^{(f)}(=$ $\sigma_{\text {diff, } 1}^{(f)}+\sigma_{\text {diff, } 2}^{(f)}$ ) and the two-nucleon diffraction, $\sigma_{\text {diff }}^{(f)}$, reaction mechanisms. Table I is for the two-proton knockout reactions from ${ }^{28} \mathrm{Mg}$ and ${ }^{54} \mathrm{Ti}$. Table II is for the two-neutron knockout reactions from ${ }^{26} \mathrm{Si},{ }^{30} \mathrm{~S}$, and ${ }^{34} \mathrm{Ar}$. The second excited state in ${ }^{24} \mathrm{Si}$ is calculated assuming it is the second $2^{+}$shell-model state. The sum of all stripping and diffraction terms and the measured values are also shown, as are the inclusive cross sections to all bound final states in the cases of the ${ }^{28} \mathrm{Mg},{ }^{26} \mathrm{Si}$, ${ }^{30} \mathrm{~S}$, and ${ }^{34} \mathrm{Ar}$ projectiles. The excited states structure in the case of the ${ }^{54} \mathrm{Ti} \rightarrow{ }^{52} \mathrm{Ca}$ reaction is not well determined by the shell model [9] and hence these excited state partial cross sections are not included here. Similarly, the recently measured ${ }^{44} \mathrm{~S} \rightarrow{ }^{42} \mathrm{Si}$ two-proton knockout reaction [8], at the $N=28$ subshell closure, poses a very interesting structural case, but one that is not well suited to the present discussion of the

TABLE I. Calculated and measured two-proton knockout reaction partial cross sections $\sigma^{(f)}$ from ${ }^{28} \mathrm{Mg}$ and ${ }^{54} \mathrm{Ti}$ on a ${ }^{9} \mathrm{Be}$ target showing their stripping, $\sigma_{\text {str }}^{(f)}$, stripping-diffraction, $\sigma_{\text {str-diff }}^{(f)}$, and diffraction, $\sigma_{\text {diff }}^{(f)}$, components. All cross sections are in mb. $R_{s}(2 N)=\sigma_{\text {expt }} / \sigma^{(f)}$ is the ratio of the experimental and the theoretical total partial cross section $\sigma^{(f)}$.

\begin{tabular}{lccccccl}
\hline \hline$J_{f}^{\pi}$ & $E(\mathrm{MeV})$ & $\sigma_{\text {str }}^{(f)}$ & $\sigma_{\text {str-diff }}^{(f)}$ & $\sigma_{\text {diff }}^{(f)}$ & $\sigma^{(f)}$ & $\sigma_{\text {expt }}[4]$ & $R_{s}(2 N)$ \\
\hline${ }^{28} \mathrm{Mg} \rightarrow{ }^{26} \mathrm{Ne}$ & $83.2 \mathrm{MeV}$ & & & & & & \\
$0^{+}$ & 0.0 & 0.63 & 0.47 & 0.09 & 1.19 & $0.70(15)$ & $0.59(13)$ \\
$2_{1}^{+}$ & 2.02 & 0.18 & 0.12 & 0.02 & 0.32 & $0.09(15)$ & $0.28(47)$ \\
$4^{+}$ & 3.50 & 0.59 & 0.37 & 0.06 & 1.02 & $0.58(9)$ & $0.57(9)$ \\
$2_{2}^{+}$ & 3.70 & 0.25 & 0.17 & 0.03 & 0.45 & $0.15(9)$ & $0.33(20)$ \\
$\mathrm{Incl.}$ & & & & & 2.98 & $1.50(10)$ & $0.50(3)$ \\
${ }^{54} \mathrm{Ti} \rightarrow{ }^{52} \mathrm{Ca}$ & $72.0 \mathrm{MeV}$ & & & & & & \\
$0^{+}$ & 0.0 & 0.21 & 0.15 & 0.03 & 0.38 & $0.21(3)$ & $0.55(8)$ \\
\hline \hline
\end{tabular}


TABLE II. As for Table I, but for the two-neutron knockout reactions from ${ }^{26} \mathrm{Si},{ }^{30} \mathrm{~S}$, and ${ }^{34} \mathrm{Ar}$, at the values of energy per nucleon indicated. The reactions are on a ${ }^{9} \mathrm{Be}$ target.

\begin{tabular}{lccccccl}
\hline \hline$J_{f}^{\pi}$ & $E(\mathrm{MeV})$ & $\sigma_{\text {str }}^{(f)}$ & $\sigma_{\text {str-diff }}^{(f)}$ & $\sigma_{\text {diff }}^{(f)}$ & $\sigma^{(f)}$ & $\sigma_{\text {expt }}[6]$ & $R_{s}(2 N)$ \\
\hline${ }^{26} \mathrm{Si} \rightarrow{ }^{24} \mathrm{Si}$ & $109.0 \mathrm{MeV}$ & & & & & & \\
$0^{+}$ & 0.0 & 0.71 & 0.50 & 0.09 & 1.30 & $0.71(9)$ & $0.55(7)$ \\
$2^{+}$ & 1.86 & 0.18 & 0.10 & 0.01 & 0.30 & $0.15(4)$ & $0.50(13)$ \\
$(4,2)^{+\mathrm{a}}$ & 3.41 & 0.18 & 0.11 & 0.02 & 0.30 & $0.14(4)$ & $0.47(13)$ \\
$\mathrm{Incl}$. & & & & & 1.90 & $1.01(10)$ & $0.53(5)$ \\
${ }^{30} \mathrm{~S} \rightarrow{ }^{28} \mathrm{~S}$ & $111.0 \mathrm{MeV}$ & & & & & & \\
$0^{+}$ & 0.0 & 0.46 & 0.32 & 0.06 & 0.84 & $0.39(8)$ & $0.46(10)$ \\
$2^{+}$ & 1.51 & 0.41 & 0.25 & 0.04 & 0.69 & $0.34(8)$ & $0.49(12)$ \\
$\mathrm{Incl}$. & & & & & 1.54 & $0.73(8)$ & $0.48(5)$ \\
${ }^{34} \mathrm{Ar} \rightarrow{ }^{32} \mathrm{Ar}$ & $110.0 \mathrm{MeV}$ & & & & & & \\
$0^{+}$ & 0.0 & 0.39 & 0.28 & 0.05 & 0.71 & $0.41(7)$ & $0.58(10)$ \\
$2^{+}$ & 1.87 & 0.18 & 0.14 & 0.03 & 0.35 & $0.07(4)$ & $0.20(11)$ \\
Incl. & & & & & 1.06 & $0.48(6)$ & $0.45(6)$ \\
\hline \hline
\end{tabular}

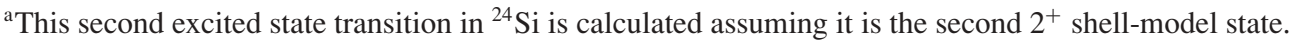

systematics that emerge from structurally better-understood cases.

We note that the sum of the stripping-diffraction $\sigma_{\text {str-diff }}^{(f)}$ and the two-nucleon diffraction, $\sigma_{\text {diff }}^{(f)}$, terms are consistently of a similar magnitude to the two-nucleon stripping cross sections, calculated here and in Ref. [5]. In the cases studied here, each of the removed nucleons is bound by approximately $15 \mathrm{MeV}$ and thus our estimated two-nucleon diffraction component is rather small, typically 5-8\%. For the removal of more weakly bound nucleons this diffraction estimate might need to be improved. However, consideration must then also be given to possible nondirect routes and cross sections to the relevant final states.

The calculated partial cross sections are consistently higher than the measured values, which can be quantified by the degree of suppression required, $R_{S}(2 N)=\sigma_{\text {expt }} / \sigma^{(f)}$. The $R_{s}(2 N)$ from each partial cross-section calculation, and the measurements, are also shown in Tables I and II. For all five reactions, the $R_{S}(2 N)$ values from the inclusive cross sections (the g.s. transition in the case of ${ }^{54} \mathrm{Ti} \rightarrow{ }^{52} \mathrm{Ca}$ ) are remarkably consistent, with value 0.5 (within one standard deviation), as shown in Fig. 2. Apart from the transition to

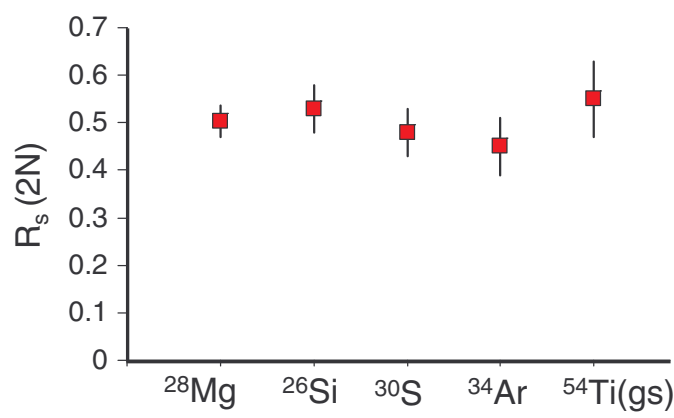

FIG. 2. (Color online) Two-nucleon suppression factors, $R_{s}(2 N)=\sigma_{\text {expt }} / \sigma_{\text {incl }}$, derived from the inclusive cross section calculations and measurements (the g.s. values in the case of ${ }^{54} \mathrm{Ti} \rightarrow{ }^{52} \mathrm{Ca}$ ) of Tables I and II. the first $2^{+}$final state in ${ }^{32} \mathrm{Ar}$, where the measured value is significantly smaller than that calculated, the same suppression is required for all the measured partial cross sections, although with less statistics and limited precision in several instances. The agreement across the four partial cross-section measurements in the case of the ${ }^{28} \mathrm{Mg} \rightarrow{ }^{26} \mathrm{Ne}\left(J^{\pi}\right)$ reaction is shown in Fig. 3. Here, the calculated cross sections for all transitions have been multiplied by the value $R_{S}(2 N)=0.50$, as deduced from the inclusive cross section, see Table I. The measured $\left(0^{+}\right)$ground-state branching ratios for ${ }^{26} \mathrm{Si},{ }^{30} \mathrm{~S}$, and ${ }^{34} \mathrm{Ar}$, and those calculated using the shell-model two-nucleon transition densities, were also found to be in excellent agreement, as was shown in Fig. 7 of Ref. [6]. These published results are not reproduced here.

Although a suppression of the shell-model transition strengths is not unexpected, it has not hitherto been quantified. The present work provides such a first, quantitative estimate of its magnitude for two-nucleon removal. The suppressions observed in nuclear-induced single-nucleon removal reactions [24,26] are entirely consistent with those observed in electron-induced single-proton knockout [27] for those

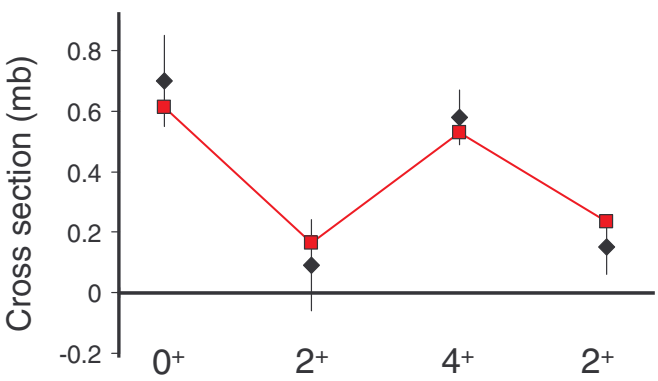

FIG. 3. (Color online) The measured [4] and calculated partial cross sections for the ${ }^{28} \mathrm{Mg} \rightarrow{ }^{26} \mathrm{Ne}\left(J^{\pi}\right)$ two-proton knockout reaction at $83.2 \mathrm{MeV}$ on a ${ }^{9} \mathrm{Be}$ target. The calculated partial cross sections, shown in Table I, have each been multiplied by $R_{s}(2 N)=0.5$, deduced from the inclusive cross section. 
systems with similar binding. These single-nucleon suppressions are understood to be a consequence of the restricted model spaces used, even in large configuration-mixed shellmodel calculations. These are unable to account quantitatively for the redistribution of single-particle strength due to short- and medium-range (tensor) nucleon-nucleon correlation effects and from longer-range correlations arising from couplings, by nucleons near the Fermi surface, to collective degrees of freedom. These phenomena are also expected to reduce the shell-model two-nucleon transition strengths. The present work thus quantifies this reduction for mid-sd-shell systems.

The ability to access removal reactions from weakly bound exotic nuclei, as is provided by the nuclear knockout mechanism, has also revealed evidence of a dependence of the observed single-nucleon suppressions, $R_{s}$, on the nucleon separation energy [20,24,28]. Any such separation energy dependence would not be revealed in the present work, where, as noted above, the two-nucleon separation energies are essentially equal for all of the systems studied. This is consistent with the observed constancy of the deduced $R_{S}(2 N)$.

\section{SUMMARY}

We have considered reactions involving the sudden, direct removal of pairs of like nucleons, of the deficient nucleon species, from exotic nuclei. We have formulated and discussed quantitatively the contributions to two-nucleon knockout reaction cross sections from events in which one nucleon is removed by the stripping mechanism and the second is removed by the diffraction mechanism. The approach used combines the eikonal reaction theory and microscopic shellmodel transition densities. The additional contributions from two-nucleon diffraction events were also estimated. For the examples considered, where each of the removed nucleons is bound by of order $15 \mathrm{MeV}$, this estimated two-nucleon diffraction component is rather small, typically only $5 \%-8 \%$ of the total two-nucleon removal cross section.

The total additional cross section from all diffraction events is of approximately the same size as that from events in which both of the nucleons are stripped. The systematics of the measured partial cross sections are in general well reproduced, but the theoretical cross sections are now larger than the measured values for ${ }^{28} \mathrm{Mg},{ }^{26} \mathrm{Si},{ }^{30} \mathrm{~S},{ }^{34} \mathrm{Ar}$, and ${ }^{54} \mathrm{Ti}$ projectiles: requiring a suppression of the shell-model transition strengths. The latter were derived from full $s d$ and $f p$-shell configuration mixed calculations. For all of the systems studied the deduced two-nucleon suppression factors $R_{s}(2 N)$ are consistent with a value of 0.5 .
[1] M. H. Macfarlane and J. P. Schiffer, in Nuclear Spectroscopy and Reactions, Part B, edited by J. Cerny, (Academic Press, New York, 1974), p. 169.

[2] C. J. G. Onderwater et al., Phys. Rev. Lett. 81, 2213 (1998).

[3] B. A. Brown and F. C. Barker, Phys. Rev. C 67, 041304(R) (2003).

[4] D. Bazin et al., Phys. Rev. Lett. 91, 012501 (2003).

[5] J. A. Tostevin, G. Podolyák, B. A. Brown, and P. G. Hansen, Phys. Rev. C 70, 064602 (2004).

[6] K. Yoneda, A. Obertelli, A. Gade, D. Bazin, B. A. Brown, C. M. Campbell, J. M. Cook, P. D. Cottle, A. Davies, C. Dinca, T. Glasmacher, P. G. Hansen, T. Hoagland, K. Kemper, J. L. Lecouey, W. F. Mueller, R. Reynolds, B. Roeder, R. Terry, J. A. Tostevin, and H. Zwahlen, Phys. Rev. C 74, 021303(R) (2006).

[7] J. A. Tostevin, J. Phys.: Conf. Ser. 49, 21 (2006).

[8] J. Fridmann, I. Wiedenhöver, A. Gade, L. T. Baby, D. Bazin, B. A. Brown, C. M. Campbell, J. M. Cook, P. D. Cottle, E. Diffenderfer, D.-C. Dinca, T. Glasmacher, P. G. Hansen, K. W. Kemper, J. L. Lecouey, W. F. Mueller, E. RodriguezVieitez, J. R. Terry, J. A. Tostevin, K. Yoneda, and H. Zwahlen, Phys. Rev. C 74, 034313 (2006).

[9] A. Gade, R. V. F. Janssens, D. Bazin, R. Broda, B. A. Brown, C. M. Campbell, M. P. Carpenter, J. M. Cook, A. N. Deacon, D.-C. Dinca, B. Fornal, S. J. Freeman, T. Glasmacher, P. G. Hansen, B. P. Kay, P. F. Mantica, W. F. Mueller, J. R. Terry,
J. A. Tostevin, and S. Zhu, Phys. Rev. C 74, 021302(R) (2006).

[10] J. A. Tostevin, AIP Conference Proceedings 819523 (2006).

[11] J. A. Tostevin, Reaction Spectroscopy at Fragmentation Beam Energies, Seventh International Conference on Radioctive Nuclear Beams (RNB7), Eur. Phys. J. A (in press).

[12] J. S. Al-Khalili, J. A. Tostevin, and I. J. Thompson, Phys. Rev. C 54, 1843 (1996).

[13] M. Hussein and K. McVoy, Nucl. Phys. A445, 124 (1985).

[14] J. A. Tostevin, Nucl. Phys. A682, 320c (2001).

[15] G. F. Bertsch, K. Hencken, and H. Esbensen, Phys. Rev. C 57, 1366 (1998).

[16] The computer code OXBASH, B. A. Brown et al., MSU-NSCL Report Number 524.

[17] B. A. Brown and B. H. Wildenthal, Annu. Rev. Nucl. Part. Sci. 38, 29 (1988).

[18] M. Honma, T. Otsuka, B. A. Brown, and T. Mizusaki, Phys. Rev. C 65, 061301(R) (2002).

[19] We point out that in Table II of Ref. [5], the column of amplitudes headed $\left[0 d_{3 / 2} 0 d_{5 / 2}\right]$ should actually read $\left[0 d_{5 / 2} 0 d_{3 / 2}\right]$. This change is typographical only. The calculations presented there interpret and use the amplitudes correctly, as those for the $\left[0 d_{5 / 2} 0 d_{3 / 2}\right]$ coupling order.

[20] A. Gade et al., Phys. Rev. Lett. 93, 042501 (2004). 
[21] B. A. Brown, Phys. Rev. C 58, 220 (1998).

[22] J. A. Tostevin, J. Phys. G 25, 735 (1999).

[23] L. Ray, Phys. Rev. C 20, 1857 (1979).

[24] P. G. Hansen and J. A. Tostevin, Annu. Rev. Nucl. Part. Sci. 53, 219 (2003).

[25] A. Ozawa et al., Nucl. Phys. A691, 599 (2001).
[26] B. A. Brown, P. G. Hansen, B. M. Sherrill, and J. A. Tostevin, Phys. Rev. C 65, 061601(R) (2002).

[27] G. J. Kramer, H. P. Blok, and L. Lapikás, Nucl. Phys. A679, 267 (2001), and references therein.

[28] J. Lee, J. A. Tostevin, B. A. Brown, F. Delaunay, W. G. Lynch, M. J. Saelim, and M. B. Tsang, Phys. Rev. C 73, 044608 (2006). 